\title{
Solving Fractional Variational Problem Via an Orthonormal Function
}

\author{
Akram Kheirabadi ${ }^{1, *}$, Asadollah Mahmoudzadeh Vaziri ${ }^{1}$, Sohrab Effati ${ }^{2}$ \\ ${ }^{1}$ Department of Mathematics, Faculty of Mathematical Science and Statistics, University of Birjand, Birjand, Iran \\ ${ }^{2}$ Department of Applied Mathematics, Faculty of Mathematical Sciences, Ferdowsi University of Mashhad, Mashhad, Iran
}

\begin{abstract}
In the present paper, a direct numerical technique for solving fractional optimal control problems based on an orthonormal wavelet, is introduced. First we approximate the involved functions by Sine-Cosine wavelet basis; then, an operational matrix is used to transform the given problem into a linear system of algebraic equations, which is easier. In fact operational matrix of Riemann-Liouville fractional integration and derivative of Sine-Cosine wavelet are employed to achieve a linear algebraic equation. The mentioned matrices are derived via hat functions. The solution of transformed system, gives us the solution of original problem. Two numerical examples are also given. Finally, the paper is ended with conclusion.
\end{abstract}

Keywords Fractional Optimal Control, Sine-Cosine Wavelet, Operational Matrix, Hat Function.

AMS 2010 subject classifications 49M99, 26A33, 49N10

DOI: $10.19139 /$ soic.v7i2.502

\section{Introduction}

In recent years, fractional calculus is one of the interesting issues that attract many scientists. Many realistic models of engineering and physical phenomena can be uttered with fractional calculus. For example they can be applied in nonlinear oscillations of earthquakes [20], fluid-dynamic traffic [21], frequency dependent damping behavior of various viscoelastic materials [4], solid mechanics [35], economics [5], signal processing [32], and control theory [10]. Niels Henrik Abel, in 1823, was probably the first to give an application of fractional calculus. Abel applied the fractional calculus in the solution of an integral equation which arises in the formulation of the problem of finding the shape of a frictionless wire lying in a vertical plane such that the time of a bead placed on the wire slides to the lowest point of the wire in the same time regardless of where the bead is placed [6].

Besides modeling, the solution techniques and their reliabilities are most important. Analytical solutions of fractional differential equations are not always available, therefore, it is important to obtain numerical solutions for these equations. The most commonly techniques proposed to solve fractional problem are Adomian Decomposition Method (ADM) [37, 29], Variational Iteration Method (VIM) [12], operational matrix method [36], homotopy analysis method [1, 13, 14], homotopy perturbation methods [18], collocation method [17], Galerkin method [28], Fractional Difference Method (FDM) [30], finite difference method [27], Fractional Differential Transform Method (FDTM) [3, 16] and power series method [31], in addition, there are some classical techniques, e.g. Laplace transform method [33]. For further study on recent papers in the area of fractional differential equations and their applications, referred to $[9,42,43]$.

A fractional optimal control problem (FOCP) is an optimal control problem in which the performance index or the differential equations in the dynamics of the system or both contain at least one fractional order derivative

\footnotetext{
${ }^{*}$ Correspondence to: Akram kheirabadi (Email: ak_kheirabadi@yahoo.com). Department of Mathematics, Faculty of Mathematical
} Science and Statistics, University of Birjand, Birjand, University blvd, Iran.

ISSN 2310-5070 (online) ISSN 2311-004X (print)

Copyright (c) 2019 International Academic Press 
term [39]. Tricaud and Chen have solved a large class of FOCPs (linear, nonlinear, time-invariant, time-variant, SISO, MIMO, state or input constrained, etc.) by converting them into a general and rational form of optimal control problem [40]. In addition to the above methods, orthogonal function method is also applicable to solve the fractional order systems and as a result, FOCPs.

Approximation by orthogonal families of basis functions is widely used in science and engineering. The main idea of applying an orthogonal basis is reduction of considered problem into a system of algebraic equations, by truncating series of orthogonal basis functions for the solution of the problem and applying operational matrix of integration and differentiation to eliminate the integral and derivative operations whenever needed, thus greatly simplifying the problem. These matrices can be uniquely determined based on the particular orthogonal functions.

The orthogonal functions are classified into three main cathegories [38], the first one is sets of piecewise constant orthogonal functions such as the Walsh functions and block pulse functions. The second one is orthogonal polynomials such as the Laguerre, Legendre and Chebyshev functions [26], and the last one is sine-cosine functions. On one hand, approximating a continuous function with piecewise constant basis functions results in a piecewise constant approximation, on the other hand, if we approximate a discontinuous function with continuous basis the resulting approximation is continuous which is not proper for modelling the discontinuities. So, neither continuous basis functions nor piecewise constant basis functions, can efficiently model both continuity and discontinuity of phenomena at the same time. In the case that the function under approximation is not analytic, wavelet functions will be more effective.

The operational matrix of fractional integrals has been derived for many types of orthogonal polynomials such as Legendre polynomials [2, 15], Jacobi polynomials [8], Laguerre polynomials [7] and so on.

In this paper, two new operational matrices are introduced and a direct method based on Sine-Cosine wavelet with their fractional integration and differentiation operational matrix is proposed to solve a FOCP and a variational problem. The main idea is to reduce the problem under consideration into a system of algebraic equations. To this end, we expand the fractional derivative of the state and control variables using the Sine-Cosine wavelet with unknown coefficients. There are many numerical methods to solve the transformed problem. The proposed method can also be applied to systems with time varying coefficients by using operational matrix of production. This matrix could easily obtained by using the sin and cos multiplication properties.

The paper is organized as follows. In next section we will give the preliminaries of fractional calculus, then in section 3 express a brief review of Hat function and the related fractional operational matrix. In section 4, we describe Sine-Cosine wavelets and its application in function approximation. In section 5, operational matrices of fractional integration and differentiation for considered wavelet is given. In section 6, the proposed method is described for solving the underlying FOCP. The proposed method is applied for solving numerical examples, in section 7. Finally, the paper is ended with conclusion.

\section{Preliminaries of fractional calculus}

Fractional order calculus deals with the non-integer order differentiation and integration. Now, we give necessary definitions of the fractional calculus theory. The most commonly used definitions for fractional integral and derivative are the Riemann-Liouville and Caputo definitions. The Riemann-Liouville fractional integration operator of a function $f$ of order $\alpha \geq 0$ is defined in [34] as

$$
\left(I^{\alpha} f\right)(t)= \begin{cases}\frac{1}{\Gamma(\alpha)} \int_{0}^{t}(t-\tau)^{\alpha-1} f(\tau) d \tau & \alpha>0, \\ f(t) & \alpha=0,\end{cases}
$$

as two properties of Riemann-Liouville fractional integration we have

$$
\begin{aligned}
& I^{\alpha} t^{k}=\frac{\Gamma(k+1)}{\Gamma(k+1+\alpha)} t^{\alpha+k} \quad k \in \mathbb{N} \cup\{0\}, t>0, \\
& I^{\alpha} I^{\beta} t^{k}=I^{\alpha+\beta} t^{k} .
\end{aligned}
$$


The fractional derivative operator of order $\alpha>0$ in the Caputo sense is defined in [34] as:

$$
D^{\alpha} f(t)=\frac{1}{\Gamma(n-\alpha)} \int_{0}^{t}(t-\tau)^{n-\alpha-1} f^{(n)}(\tau) d \tau=I^{n-\alpha} f^{(n)}(t) \quad n-1<\alpha \leq n .
$$

Two useful relation between the Riemann-Liouville and Caputo operators is as follow

$$
\begin{aligned}
& D^{\alpha} I^{\alpha} f(t)=f(t), \\
& I^{\alpha} D^{\alpha} f(t)=f(t)-\sum_{0}^{n-1} f^{k}(0) \frac{t^{k}}{k !} \quad t>0, n-1<\alpha \leq n .
\end{aligned}
$$

\section{Review of Hat functions and the related fractional operational matrix}

In this section, we introduce Hat functions (HFs) and its operational matrix of fractional integration.

\subsection{Definition of HFs}

A $\hat{m}$ - set of HFs basis functions is defined by Tripathi et al. [41] as follows:

$$
\begin{aligned}
\phi_{0}(t) & = \begin{cases}\frac{h-t}{h} \quad 0 \leq t<h, \\
0 \quad \text { O.W. }\end{cases} \\
\phi_{i}(t) & = \begin{cases}\frac{t-(i-1) h}{h} & (i-1) h \leq t<i h, \\
\frac{(i+1) h-t}{h} & i h \leq t<(i+1) h \quad i=1,2, \cdots, \hat{m}-2, \\
0 & O . W .\end{cases} \\
\phi_{\hat{m}-1}(t) & = \begin{cases}\frac{t-(1-h)}{h} & 1-h \leq t \leq 1, \\
0 & O . W .\end{cases}
\end{aligned}
$$

where $h=\frac{1}{\hat{m}-1}$. An arbitrary function like $f(t) \in L^{2}[0,1]$ can be expanded by HFs as:

$$
f(t)=\sum_{i=0}^{\hat{m}-1} f_{i} \phi_{i}(t)=F^{T} \Phi(t)=\Phi^{T}(t) F
$$

where

$$
F=\left[f_{0}, f_{1}, \cdots, F_{\hat{m}-1}\right]^{T}, \quad \Phi=\left[\phi_{0}, \phi_{1}, \cdots, \phi_{\hat{m}-1}\right]^{T} .
$$

An important property of HFs in approximating the function $f(t)$ is that the coefficients $f_{i}$ in the above equation are stated by:

$$
f_{i}=f(i h), \quad i=0,1, \cdots, \hat{m}-1 .
$$

\subsection{Operational matrix of fractional integration for $\mathrm{HFs}$}

Operational matrix of fractional integration of order $\alpha$ for HFs, which given in [41] is as:

$$
\left(I^{\alpha} \Phi\right)(t) \simeq Q^{\alpha} \Phi(t)
$$


where

$$
Q^{\alpha}=\frac{h^{\alpha}}{\Gamma(\alpha+2)}\left(\begin{array}{cccccc}
0 & \zeta_{1} & \zeta_{2} & \cdots & \zeta_{\hat{m}-2} & \zeta_{\hat{m}-1} \\
0 & 1 & \xi_{1} & \cdots & \xi_{\hat{m}-3} & \xi_{\hat{m}-2} \\
0 & 0 & 1 & \cdots & \xi_{\hat{m}-4} & \xi_{\hat{m}-3} \\
\vdots & \vdots & \vdots & \cdots & \vdots & \vdots \\
0 & 0 & 0 & 0 & 1 & \xi_{1} \\
0 & 0 & 0 & 0 & 0 & 1
\end{array}\right)
$$

and

$$
\begin{array}{ll}
\zeta_{i}=i^{\alpha}(\alpha-i+1)+(i-1)^{\alpha+1}, & i=1,2, \cdots, \hat{m}-1 \\
\xi_{i}=(i+1)^{\alpha+1}-2 i^{\alpha+1}+(i-1)^{\alpha+1}, & i=1,2, \cdots, \hat{m}-2
\end{array}
$$

\section{Description of Sine-Cosine wavelets and its application in function approximation}

Wavelets have been very successful in approximate solution of different types of systems. They constitute a family of functions constructed from dilation and translation of a single function called the mother wavelet. When the dilation parameter $a$ and the translation parameter $b$ vary continuously, we have the following family of continuous wavelets [19].

$$
\psi_{a, b}=|a|^{-\frac{1}{2}} \psi\left(\frac{t-b}{a}\right) \quad a, b \in \mathbb{R}, a \neq 0,
$$

if we restrict the parameters $a$ and $b$ to discrete values $a=a_{0}^{-k}, b=n b_{0} a_{0}^{-k}$, where $a_{0}>1, b_{0}>0, n$ and $k$ are positive integers, we have the following family of discrete wavelets:

$$
\psi_{k, n}=\left|a_{0}\right|^{\frac{k}{2}} \psi\left(a_{0}^{K} t-n b_{0}\right)
$$

which are a wavelet basis for $L^{2}(\mathbb{R})$. Sine-Cosine wavelets are defined as follows [23]

$$
\psi_{n, m}(t)= \begin{cases}2^{\frac{k+1}{2}} f_{m}\left(2^{k} t-n\right) & \frac{n}{2^{k}} \leq t<\frac{n+1}{2^{k}}, \\ 0 & O . W .\end{cases}
$$

with

$$
f_{m}(t)= \begin{cases}\frac{1}{\sqrt{2}} & m=0, \\ \cos (2 m \pi t) & m=1,2, \cdots, l, \\ \sin (2(m-l) \pi t) & m=l+1, \cdots, 2 l,\end{cases}
$$

$n=0,1, \cdots, 2^{k}-1, k=0,1, \cdots$ and $l$ is any positive integer. A function $f(t) \in L^{2}[0,1)$ can be approximated as:

$$
f(t)=\sum_{n=0}^{2^{k}-1} \sum_{m=0}^{2 l} c_{n, m} \psi_{n, m}(t)=C^{T} \Psi(t)=\Psi^{T}(t) C,
$$

where $c_{n, m}=\left\langle f(t), \psi_{n, m}\right\rangle$ and $\langle.,$.$\rangle denotes the inner product as:$

$$
c_{n, m}=\int_{-\infty}^{+\infty} f(t) \psi_{n, m}(t) d t
$$

$\Psi(t)$ represent the vector of considered wavelet. $C$ and $\Psi(t)$ are $2^{k}(2 l+1) \times 1$ matrices which are given by:

$$
\begin{aligned}
C^{T} & =\left[c_{0,0}, c_{0,1}, \cdots, c_{0,2 l}, c_{1,0}, \cdots, c_{1,2 l}, \cdots, c_{2^{k}-1,0}, \cdots, c_{2^{k}-1,2 l}\right], \\
\Psi^{T} & =\left[\psi_{0,0}, \psi_{0,1}, \cdots, \psi_{0,2 l}, \psi_{1,0}, \cdots, \psi_{1,2 l}, \cdots, \psi_{2^{k}-1,0}, \cdots, \psi_{2^{k}-1,2 l}\right] .
\end{aligned}
$$




\section{Operational matrix of fractional calculus for Sine-Cosine wavelet}

In this section, we derive the operational matrix of fractional derivative for the considered wavelet using the operational matrix of fractional integration for HFs.

\subsection{Express $\Psi(t)$ in terms of HFs}

Each $\psi_{n, m}(t)$ as a function, can be expanded in terms of HFs function, thus for $\Psi(t)$ we will have

$$
\Psi(t) \simeq \Phi_{\hat{m} \times \hat{m}} \Phi(t)
$$

In the above equation $\hat{m}=2^{k}(2 l+1)$ and $\Phi_{\hat{m} \times \hat{m}}$ obtain as follow. We choose $h=\frac{1}{\hat{m}-1}$, then by considering the property which is given in Eq. (6) we have

$$
\begin{aligned}
& \psi_{n, m}(t)=\sum_{i=0}^{\hat{m}-1} c_{n, m}^{i} \phi_{i}(t)=C_{n, m} \Phi(t), \\
& c_{n, m}^{i}=\psi_{n, m}(i h), \\
& C_{n, m}=\left[c_{n, m}^{0}, c_{n, m}^{1}, \cdots, c_{n, m}^{\hat{m}-1}\right]=\left[\psi_{n, m}(0), \psi_{n, m}(h), \cdots, \psi_{n, m}(1)\right],
\end{aligned}
$$

therefore

$$
\psi_{n, m}(t)=\left[\psi_{n, m}(0), \psi_{n, m}(h), \cdots, \psi_{n, m}(1)\right] \Phi(t)
$$

The vector $C_{n, m}$ represent a row of matrix $\Phi_{\hat{m} \times \hat{m}}$.

\subsection{Operational matrix of fractional integration and derivative for Sine-Cosine wavelet}

Suppose $\Psi(t)$ be the vector defined in (9), then, fractional integration of order $\alpha>0$ in the Riemann-Liouville sense of this vector can be expressed as

$$
\left(I^{\alpha} \Psi\right)(t) \simeq P^{\alpha} \Psi(t)
$$

where $P^{\alpha}$ is the operational matrix of fractional integration. By considering Eq. (11), $P^{\alpha}$ calculated as follows

$$
I^{\alpha} \Psi(t) \simeq I^{\alpha} \Phi_{\hat{m} \times \hat{m}} \Phi(t)=\Phi_{\hat{m} \times \hat{m}} I^{\alpha} \Phi(t) \simeq \Phi_{\hat{m} \times \hat{m}} Q^{\alpha} \Phi(t) .
$$

Using Eqs. (13) and (14) we obtain

$$
P^{\alpha} \Psi(t) \simeq P^{\alpha} \Phi_{\hat{m} \times \hat{m}} \Phi(t)=\Phi_{\hat{m} \times \hat{m}} Q^{\alpha} \Phi(t) \Rightarrow P^{\alpha}=\Phi_{\hat{m} \times \hat{m}} Q^{\alpha} \Phi_{\hat{m} \times \hat{m}}^{-1} .
$$

Now we calculate operational matrix of derivative using $P^{\alpha}$, suppose that $x(t) \simeq X^{T} \Psi(t)$ then we have

$$
\begin{aligned}
& D^{\alpha} f(t)=I^{n-\alpha} f^{(n)}(t) \quad n-1<\alpha \leq n, \quad n \in \mathbf{N}, \\
& D^{\alpha} x(t) \simeq D^{\alpha} X^{T} \Psi(t)=X^{T} D^{\alpha} \Psi(t)=X^{T} I^{n-\alpha} \Psi^{(n)}(t),
\end{aligned}
$$

for $\alpha \in(0,1]$ we have $n=1$ thus

$$
D^{\alpha} x(t) \simeq X^{T} I^{1-\alpha} D \Psi(t)=X^{T} D I^{1-\alpha} \Psi(t)=X^{T} D \Phi_{\hat{m} \times \hat{m}} Q^{1-\alpha} \Phi_{\hat{m} \times \hat{m}}^{-1} \Psi(t)
$$


where $\mathrm{D}$ is the operational matrix of derivative for $\Psi(t)$ which defined as $D=\operatorname{diag}(W, W, \cdots, W)$, which is a $2^{k}(2 l+1) \times 2^{k}(2 l+1)$ matrix and $W$ is of size $(2 l+1) \times(2 l+1)$

$$
W=2^{k+1} \pi\left(\begin{array}{ccccccccc}
0 & 0 & 0 & \cdots & 0 & 0 & 0 & \cdots & 0 \\
0 & 0 & 0 & \cdots & -1 & 0 & 0 & \cdots & 0 \\
0 & 0 & 0 & \cdots & 0 & -2 & 0 & \cdots & 0 \\
\vdots & \vdots & \vdots & \ddots & \vdots & \vdots & \vdots & \ddots & \vdots \\
0 & 0 & 0 & \cdots & 0 & 0 & 0 & \cdots & -l \\
0 & 1 & 0 & \cdots & 0 & 0 & 0 & \cdots & 0 \\
0 & 0 & 2 & \cdots & 0 & 0 & 0 & \cdots & 0 \\
\vdots & \vdots & \vdots & \ddots & \vdots & \vdots & \vdots & \ddots & \vdots \\
0 & 0 & 0 & \cdots & l & 0 & 0 & \cdots & 0
\end{array}\right)_{(2 l+1) \times(2 l+1)}
$$

\section{Solution of fractional optimal control problem by Sine-Cosine operational matrix}

Consider the fractional optimal control problem with quadratic performance index

$$
\begin{aligned}
\min & J=\frac{1}{2} X^{T}(1) S X(1)+\frac{1}{2} \int_{0}^{1}\left(X^{T}(t) Q X(t)+U^{T}(t) R U(t)\right) d t, \\
\text { st. } & D^{\alpha} X(t)=A X(t)+B U(t) \quad 0<\alpha \leq 1, \\
& X(0)=X_{0},
\end{aligned}
$$

where $\mathrm{A}$ and $\mathrm{B}$ are constant matrices with the appropriate dimensions, also in cost functional, $\mathrm{S}$ and $\mathrm{Q}$ are symmetric positive semi- definite matrices and $\mathrm{R}$ is a symmetric positive definite matrix.

$$
\begin{aligned}
& X(t)=\left[x_{1}(t), x_{2}(t), \cdots, x_{s}(t)\right]^{T} \quad x_{i}(t)=\Psi^{T}(t) X_{i}=X_{i}^{T} \Psi(t), \\
& X(t)=\tilde{\Psi}_{s}^{T}(t) X \quad X=\left[X_{1}^{T}, X_{2}^{T}, \cdots, X_{s}^{T}\right]^{T} \quad \tilde{\Psi}_{s}(t)=I_{s} \otimes \Psi(t), \\
& U(t)=\left[u_{1}(t), u_{2}(t), \cdots, u_{q}(t)\right]^{T} \quad u_{i}(t)=\Psi^{T}(t) U_{i}=U_{i}^{T} \Psi(t), \\
& U(t)=\tilde{\Psi}_{q}^{T}(t) U \quad U=\left[U_{1}^{T}, U_{2}^{T}, \cdots, U_{q}^{T}\right]^{T} \quad \tilde{\Psi}_{q}(t)=I_{q} \otimes \Psi(t),
\end{aligned}
$$

thus

$$
J=\frac{1}{2} X^{T} \tilde{\Psi}_{s}(1) S \tilde{\Psi}_{s}^{T}(1) X+\frac{1}{2} \int_{0}^{1}\left[X^{T} \tilde{\Psi}_{s} Q \tilde{\Psi}_{s}^{T} X+U^{T} \tilde{\Psi}_{q} R \tilde{\Psi}_{q}^{T} U\right] d t
$$

The considered wavelet is orthonormal, it means $\int_{0}^{1} \Psi^{T}(t) \Psi(t) d t=I$, thus we can rewrite (21) as follows

$$
J(X, U)=\frac{1}{2} X^{T}\left[S \otimes \tilde{\Psi}_{s}(1) \tilde{\Psi}_{s}^{T}(1)\right] X+\frac{1}{2}\left[X^{T}(Q \otimes I) X+U^{T}(R \otimes I) U\right],
$$

similarly, we use (17) and (20) for the dynamic system in (19)

$$
\begin{aligned}
X(t) & =X^{T} I_{s} \otimes \Psi(t)=\left(I_{s} \otimes \Psi^{T}(t)\right) X, \\
D^{\alpha} X(t) & =I^{1-\alpha} X^{\prime}(t)=I^{1-\alpha}\left(X^{T}\left(I_{s} \otimes \Psi(t)\right)\right)^{\prime}=X^{T} I^{1-\alpha}\left(I_{s} \otimes(D \Psi(t))\right)=X^{T} I_{s} \otimes\left(I^{1-\alpha} D \Psi(t)\right) \\
& =X^{T} I_{s} \otimes\left(D I^{1-\alpha} \Psi(t)\right)=X^{T} I_{s} \otimes\left(D \Phi_{\hat{m} \times \hat{m}} Q^{1-\alpha} \Phi_{\hat{m} \times \hat{m}}^{-1} \Psi(t)\right),
\end{aligned}
$$

using dynamic system of (19) and (23), we set

$$
\begin{aligned}
& R(t)=X^{T} I_{s} \otimes\left(D \Phi_{\hat{m} \times \hat{m}} Q^{1-\alpha} \Phi_{\hat{m} \times \hat{m}}^{-1}\right) \Psi(t)-A X^{T} I_{s} \otimes \Psi(t)-B U^{T} I_{q} \otimes \Psi(t), \\
& R(t)=\left[X^{T} I_{s} \otimes\left(D \Phi_{\hat{m} \times \hat{m}} Q^{1-\alpha} \Phi_{\hat{m} \times \hat{m}}^{-1}\right)-A X^{T} I_{s} \otimes I_{2^{k}(2 l+1)}-B U^{T} I_{q} \otimes I_{2^{k}(2 l+1)}\right] \Psi(t) .
\end{aligned}
$$


As in a typical tau method [11] we generate $2^{k}(2 l+1)-1$ linear equations by applying

$$
\left\langle R(t), \psi_{n, m}(t)\right\rangle=\int_{0}^{1} R(t) \cdot \psi_{n, m}(t) d t=0,
$$

also, for boundary value we have

$$
X(0)=X^{T} \tilde{\Psi}(0)=X_{0} .
$$

Eqs. (24) and (25) generate $2^{k}(2 l+1)$ set of linear equations. These linear equations can be solved for unknown coefficients of the vectors $X^{T}$ and $U^{T}$. Consequently, $X(t)$ and $U(t)$ can be calculated.

\section{Illustrative example}

We applied the method presented in this paper and solved the undergoing examples.

\section{Example 1}

Consider the following time invariant FOCP [25]

$$
\begin{aligned}
\min & J=\frac{1}{2} \int_{0}^{1}\left(x^{2}(t)+u^{2}(t)\right) d t, \\
& D^{\alpha} x(t)=-x(t)+u(t), \\
& x(0)=1 .
\end{aligned}
$$

We want to find a control variable $u(t)$ which minimizes the quadratic performance index $J$. This problem is solved by proposed method, the numerical value obtained for $\mathrm{J}$ is 0.1932 , which is close to the exact solution in the case $\alpha=1$ (0.1929).

Example 2

Consider the following functional

$$
\min \quad J(t)=\int_{0}^{1}\left[\left(D^{\alpha} x(t)\right)^{2}+t D^{\alpha} x(t)\right] d t,
$$

and the boundary conditions $x(0)=0$ and $x(1)$ is unspecified.

For solving the above problem we use the undergoing relation

$$
D^{\alpha} x(t)=C^{T} \Psi(t) \Rightarrow x(t)=C^{T} P^{\alpha} \Psi(t)+x(0),
$$

we expand $t$ in terms of considered wavelet as $t=d^{T} \Psi(t)$ where $d$ is as follows

$$
\begin{gathered}
d=2^{\left(\frac{-2-3 k}{2}\right)}\left[1,0, \cdots, 0,-\frac{\sqrt{2}}{\pi}, \cdots,-\frac{\sqrt{2}}{l \pi} \mid 3,0, \cdots, 0,-\frac{\sqrt{2}}{\pi}, \cdots,-\frac{\sqrt{2}}{l \pi}\right. \\
\left.|\cdots| 2^{k+1}-1,0, \cdots, 0,-\frac{\sqrt{2}}{\pi}, \cdots,-\frac{\sqrt{2}}{l \pi}\right]^{T}
\end{gathered}
$$

because $x(1)$ is unspecified we have

$$
2 D^{\alpha} x(t)+\left.t\right|_{t=1}=0 \Rightarrow C^{T} \Psi(1)=-\frac{1}{2}
$$

by substituting Eqs.(28) and (29) in (27) we get

$$
J(t)=\int_{0}^{1}\left[C^{T} \Psi(t) \Psi(t)^{T} C+d^{T} \Psi(t) \Psi(t)^{T} C\right] d t=C^{T} C+d^{t} C,
$$

now we have to minimize quadratic function (31) subject to constraint (30). The exact value for $\mathrm{J}$ is -0.0833 [22] and the value obtian via above method is -0.08328635 , which is acceptable. 


\section{Conclusion}

In this paper, we use a direct numerical method for fractional problems based on two new operational matrices of fractional integration and differentiation. The procedure of constructing these matrices is summarized. This matrices are utilized along with tau method in order to reduce the fractional differential equations into the algebraic equations which can be efficiently solved. The proposed approach is computationally simple. Two examples are given to show the efficiency of method. The result obtained in this paper is more acceptable in comparison with [24], where the fractional operational matrices of the Sine-Cosine wavelet are obtained using block-pulse functions. The obtained matrices can also be used to solve fractional optimal control with delay or multi-delay.

\section{REFERENCES}

1. T. Akbarian, and M. Keyanpour A new approach to the numerical solution of fractional order optimal control problems Applications and Applied Mathematics, vol. 8, no. 2, pp. 523-534, 2013.

2. M. H. Akrami, M. H. Atabakzadeh, and G. H. Erjaee, The operational matrix of fractional integration for shifted Legendre polynomials, Iranian Journal of Science and Technology, vol. 37, no. 4, pp. 439-444, 2013.

3. A. Arikoglu, and I. Ozkol, Solution of fractional integro-differential equations by using fractional differential transform method, Chaos, Solitons \& Fractals, vol. 40, no. 2, pp. 521-529, 2009.

4. R. L. Bagley, and P. J. Torvik, A theoretical basis for the application of fractional calculus to viscoelasticity, Journal of Rheology, vol. 27, no. 3, pp. 201-210, 1983.

5. R. T. Baillie, Long memory processes and fractional integration in econometrics, Journal of Econometrics, vol. 73, no.1, pp. 5-59, 1996.

6. N. R. Bastos, Calculus of variations involving Caputo-Fabrizio fractional differentiation, Statistics, Optimization and Information Computing, vol. 6, no. 1, pp. 12-21, 2018.

7. A. H. Bhrawy, D. Baleanu, and L. Assas, Efficient generalized Laguerre spectral methods for solving multi-term fractional differential equations on the half line, Journal of Vibration and Control, Vol. 20, no. 7, pp. 973-985, 2014.

8. A. H. Bhrawy, E. H. Doha, D. Baleanu, and S. S. Ezz-Eldien, A spectral tau algorithm based on Jacobi operational matrix for numerical solution of time fractional diffusion-wave equations, Journal of Computational Physics, vol. 293, pp. 142-156, 2015.

9. A. Biswas, A. H. Bhrawy, M. A. Abdelkawy, A. A. Alshaery, and E. M. Hilal, Symbolic computation of some nonlinear fractional differential equations, Romanian Journal of Physics, vol.59, no. 5-6, pp. 433-442, 2014.

10. G. W. Bohannan, Analog fractional order controller in temperature and motor control applications, Journal of Vibration and Control, vol. 14, no. 9-10, pp. 1487-1498, 2008.

11. C. Canuto, M. Y. Hussaini, A. Quarteroni, and T. A. Zang, Spectral Methods in Fluid Dynamic, Springer Berlin Heidelberg, 2012.

12. S. Das, Analytical solution of a fractional diffusion equation by variational iteration method, Computers \& Mathematics with Applications, vol. 57, no. 3, pp. 483-487, 2009.

13. M. Dehghan, J. Manafian, and A. Saadatmandi, Solving nonlinear fractional partial differential equations using the homotopy analysis method, Numerical Methods for Partial Differential Equations: An International Journal, vol. 26, no. 2, pp. 448-479, 2010.

14. M. Dehghan, J. Manafian Herris, and A. Saadatmandi, The Solution of the linear fractional partial differential equations using the homotopy analysis method, Zeitschrift für Naturforschung-A, vol. 65, no. 11, pp. 935-949, 2010.

15. E. H. Doha, A. H. Bhrawy, and S. S. Ezz-Eldien, An efficient Legendre spectral tau matrix formulation for solving fractional sub-diffusion and reaction sub-diffusion equations, Journal of Computational and Nonlinear Dynamics, vol. 10, no. 2, pp. 021019, 2015.

16. V. S. Erturk, and S. Momani, Solving systems of fractional differential equations using differential transform method, Journal of Computational and Applied Mathematics, vol. 2015, no. 1, pp. 142-159, 2008.

17. M. R. Eslahchi, M. Dehgh, and M. Parvizi, Application of the collocation method for solving nonlinear fractional integro-differential equations, Journal of Computational and Applied Mathematics, vol. 257, no.1, pp. 105-128, 2014.

18. B. Ghazanfari, and A. Sepahvandzadeh, Homotopy perturbation method for solving fractional Bratu-type equation, Journal of Mathematical Modeling, vol. 2, no. 2, pp. 143-155, 2015.

19. J. S. Gu, and W. S. Jiang, The Haar wavelets operational matrix of integration, International Journal of Systems Science, vol. 27, no. 7, pp. 623-628, 1996.

20. J. He, Nonlinear oscillation with fractional derivative and its applications, International Conference on Vibrating Engineering, Dalian, China, vol. 98, pp. 288-291, 1998 .

21. J. He, Some applications of nonlinear fractional differential equations and their approximations, Bulletin of Science, Technology \& Society, vol. 15 , no. 2, pp. 86-90, 1999.

22. C. H. Hsiao, Haar wavelet direct method for solving variational problem, Mathematics and Computers in Simulation, vol. 64, no. 5, pp. 569-585, 2004.

23. M. T. Kajani, M. Ghasemi, and E. Babolian, Numerical solution of linear integro-differential equation by using SineCCosine wavelets, Applied Mathematics and Computation, vol. 180, no. 2, pp. 569-574, 2006.

24. A. Kheirabadi, A. Mahmoudzadeh Vaziri, and S. Effati, A new approach for solving optimal control problem by using orthogonal function, Springer, International Conference on Management Science and Engineering Management, pp. 223-232, 2017.

25. A. Lotfi, M. Dehghan, and S. A. Yousefi, A numerical technique for solving fractional optimal control problems, Computers \& Mathematics with Applications, vol. 62, no. 3, pp. 1055-1067, 2011. 
26. I. Malmir, Optimal control of linear time-varying systems with state and input delays by Chebyshev wavelets, Statistics, Optimization and Information Computing, vol. 5, no. 4, pp. 302-324, 2017.

27. M. Meerschaert, and C. Tadjeran, Finite difference approximations for two-sided space-fractional partial differential equations, Applied Numerical Mathematics, vol. 56, no. 1, pp. 80-90, 2006.

28. F. Mohammadi, Wavelet Galerkin method for solving stochastic fracthional differential equations, Journal of Fractional Calculus and Applications, vol. 7, no. 1, pp. 73-86, 2016.

29. S. Momani, and Z. Odibat, Numerical approach to differential equations of fractional order, Journal of Computational and Applied Mathematics, vol. 207, no. 1, pp. 96-110, 2007.

30. S. Momani, and Z. Odibat, Numerical comparison of methods for solving linear differential equations of fractional order, Chaos Solitons \& Fractals, vol. 31, no. 5, pp. 1248-1255, 2007.

31. Z. Odibat, and N. Shawagfeh, Generalized Taylors formula, Applied Mathematics and Computation, vol. 186, no. 1, pp. 286-293, 2007.

32. R. Panda, and M. Dash, Fractional generalized splines and signal processing, Signal Process, vol. 86, no. 9, pp. $2340-2350,2006$.

33. I. Podlubny, The Laplace transform method for linear differential equations of the fractional order, arXiv preprint funct-an/9710 0 05, 1997.

34. I. Podlubny, Fractional differential equations: an introduction to fractional derivatives, fractional differential equations, to methods of their solution and some of their applications, Elsevier, vol. 198, 1998.

35. Y. A. Rossikhin, and M. V. Shitikova, Applications of fractional calculus to dynamic problems of linear and nonlinear hereditary mechanics of solids, Applied Mechanics Reviews, vol. 50, no. 1, pp. 15-67, 1997.

36. A. Saadatmandi, and M. Dehghan, A new operational matrix for solving fractional order differential equations, Computers \& Mathematics with Applications, vol. 59, no. 3, pp. 1326-1336, 2010.

37. N. T. Shawagfeh, Analytical approximate solutions for nonlinear fractional differential equations, Applied Mathematics and Computation, vol. 131, no. 2, pp. 517-529, 2002.

38. S. Sohrabi, Comparision Chebyshev wavelets method with BPFS method for solving Abels integral equation, Ain Shams Engineering Journal, vol. 2, no. 3-4, pp. 249-254, 2011.

39. X. W. Tangpong, and O. Agrawal, Fractional optimal control of a continuum system, Journal of Vibration and Acoustics, vol. 131, no. 2, p. $021012,2009$.

40. C. Tricaud, and Y. Chen, An approximation method for numerically solving fractional order optimal control problems of general form, Computers \& Mathematics with Applications, vol. 59, pp. 1644-1655, 2010.

41. M. P. Tripathi, V. K. Baranwal, R. K. Pandey, and O. P. Singh, A new numerical algorithm to solve fractional differential equations based on operational matrix of generalized hat functions, Communications in Nonlinear Science and Numerical Simulation, vol. 18 , no. 6, pp. 1327-1340, 2013.

42. G. W. Wang, and T. Z. Xu, The modified fractional sub-equation method and its applications to nonlinear fractional partial differential equations, Romanian Journal of Physics, vol. 59, no. 7-8, pp. 636-645, 2014.

43. X. J. Yang, D. Baleanu, Y. Khan, and S. T. Mohyud-Din, Local fractional variational iteration method for diffusion and wave equations on Cantor sets Romanian Journal of Physics, vol.59, no. 1-2, pp. 36-48, 2014. 